\title{
NEURONAL INFORMATION PROCESSING
}

\author{
From Biological Data to \\ Modelling and Applications
}




\title{
SERIES IN MATHEMATICAL BIOLOGY AND MEDICINE
}

\author{
Series Editors: P. M. Auger and R. V. Jean
}

Published

Vol. 1: Stochastic Models of Tumor Latency and their Biostatistical Applications
A. Yu Yakovlev and A. D. Tsodikov

Vol. 2: Volterra-Hamilton Models in the Ecology and Evolution of Colonial Organisms P. L. Antonelli and R. H. Bradbury

Vol. 4: Symmetry in Plants

Ed. by R. V. Jean and D. Barabé

Vol. 5: Computational Medicine, Public Health and Biotechnology: Building a Man in the Machine - Proceedings of the First World Congress

Vols. 1 \& 3-ed. by $M$. Witten

Vol. 2 - ed. by $D$. Joan and $M$. Witten

Vol. 6: Advances in Mathematical Population Dynamics - Molecules,Cells and Man Proceedings of the 4th International Conference on Mathematical Population Dynamics Ed. by O. Arino, D. Axelrod and M. Kimmel

Forthcoming

Vol. 3: The Hierarchical Genome and Differentiation Waves: Novel Unification of Development, Genetics and Evolution R. Gordon 


\title{
NEURONAL INFORMATION PROCESSING
}

\section{From Biological Data to Modelling and Applications}

\author{
Cargèse, France 30 June - 12 July 1997
}

\author{
Editors \\ G. Burdet \\ P. Combe \\ O. Parodi
}

Centre de Physique Théorique

CNRS-Luminy, France 


\section{Published by}

World Scientific Publishing Co. Pte. Ltd.

P O Box 128, Farrer Road, Singapore 912805

USA office: Suite 1B, 1060 Main Street, River Edge, NJ 07661

UK office: 57 Shelton Street, Covent Garden, London WC2H 9HE

\section{British Library Cataloguing-in-Publication Data}

A catalogue record for this book is available from the British Library.

\section{NEURONAL INFORMATION PROCESSING \\ From Biological Data to Modelling and Applications \\ Series in Mathematical Biology and Medicine, Vol. 7}

Copyright () 1999 by World Scientific Publishing Co. Pte. Ltd.

All rights reserved. This book, or parts thereof, may not be reproduced in any form or by any means, electronic or mechanical, including photocopying, recording or any information storage and retrieval system now known or to be invented, without written permission from the Publisher.

For photocopying of material in this volume, please pay a copying fee through the Copyright Clearance Center, Inc., 222 Rosewood Drive, Danvers, MA 01923, USA. In this case permission to photocopy is not required from the publisher.

ISBN 981-02-3826-6

Printed in Singapore. 


\section{TRAITEMENT NEURONAL DE L'INFORMATION des données biologiques à la modélisation et aux applications}

Le présent document a été établi en exécution du contrat $N^{\circ} 96 \mathrm{~A} 0182$ passé par la Direction de la Recherche et de la Technologie - Direction Scientifique section soutien à la recherche. 
This page is intentionally left blank 


\section{FOREWORD}

For the last ten years, statistical mechanics and dynamics of neuronal automata have been extensively studied. Most of the work was based on classical modelling of neurons. Recent developments in Neurosciences have, however, considerably modified our knowledge of both the operating modes of neurons and the information processing in the cortex.

Multi-unit recordings have allowed precise temporal correlations to be detected, within temporal windows of the order of a millisecond. In the same time, oscillations corresponding to a quasi-periodic spike-firing, synchronized over several visual cortical areas, have been observed with anaesthesied cats and with monkeys. Last but not least, recent work on the neuronal operating modes have emphasized the role played by the dendritic arborization.

These developments have deeply modified our ideas on how information is coded by neurons. The coding scheme seems now to rely rather on precise spatio-temporal patterns than on spike rates, at the cortical level at least. This leads us to consider, for information processing, new models which would proceed, e.g., from a synchronous detection of correlated spike firing, and would therefore be particularly robust against noise. Such models might lead to original technical applications for information processing and control.

Further developments in this field may be of major importance for our understanding of the basic mechanism of perception and cognition. They may also may lead to new concepts in applications directed towards artificial perception and pattern recognition. Up to now, artificial systems for pattern recognition are far to reach the standards of human vision. Systems based on a temporal coding by spikes may now be expected to bring major improvements in this field.

The aim of the school was to bring to students and people engaged in both applied and basic research a high level teaching in every involved branch (Neurosciences, Physics, Mathematics, Information and Control Theory) and to encourage further exchanges. Four lectures of ninety minutes have been daily given for ten days and have been completed by locally organized poster sessions. 
The school was organized as a Cargèse Summer School from 1997 June 30 to July 12, at the Institut d'Études Scientifiques de Cargèse, in Corsica. The institute is a joint unit (UMS 820) of Centre National de la Recherche Scientifique, Université de Corte and Université de-Nice-Sophia-Antipolis. It is our pleasure to express our gratitude to the three institutions for their hospitality, to Elisabeth Dubois-Violette, Director of the Institute, for her constant and friendly help, and to Annette Touchant, Secretary of the Institute, as well as to the whole Institute staff, for the quality of the reception and for their heartly welcome, which was for a good part in the spirit and the success of the school.

The school was, for a large part, supported by Formation Permanente du Centre National de la Recherche Scientifique. This support was decisive at a time when it was not clear whether we should be able to hold the school or not. It was our hope that the school might be useful to a large number of CNRS staff and scientists, and it is our regret that so few were able to come. All French young students came from CNRS institutes, and we hope that the knowledge they brought back from Cargèse will be of some help for them and for their colleagues in their future work.

We are greatly indebted to Direction Scientifique du Département Sciences pour l'Ingnieur du Centre National de la Recherche Scientifique who spontaneously decided to support the school.

The support of Direction des Recherches et Études Techniques - Direction Générale de l'Armement of the French Ministère de la Défense was very important for us. Let its director find here the expression of our gratitude.

Last, but not least, it is a great pleasure for us to acknowledge Collectivité Territoriale Corse, its President and its Service de l'Enseignement et de la Recherche for their help and support. We are sure to interpretate the general feeling in telling how splendid we have found Corsica, and how friendly, Corsicans. The magic island has also contributed for a large part to the spirit and success of the school.

Let us also thank every lecturer for the quality of their lectures and for their participation to the School. We hoped we should be able to publish every lecture, and we regret that some of the lecturers had not the opportunity to send us their manuscripts. We are greatly indebted to the lecturers who have sent theirs, and we hope that, even partial, these Proceedings will be of some use to those who attended the School, as well as to those who did not.

Financial support and good lectures are an essential, but not sufficient, condition for success. The spirit of the school was brought up by the participation of everyone to discussions and activities. Let us, therefore, thank every student for his or her participation. The poster session was a great moment, and we wish that it has induced some collaborations. Our hope is that the 
School has opened new perspectives and resulted in new interdisciplinary and international cooperation.

Finally the editors would like to thank the many people who made the institute and volume possible. Of especially direct help to ourselves were JeanBruno Erismann, Denis Patrat, Michèle Rossignol and Françoise Smagghe from the Centre de Physique Théorique de Marseille.

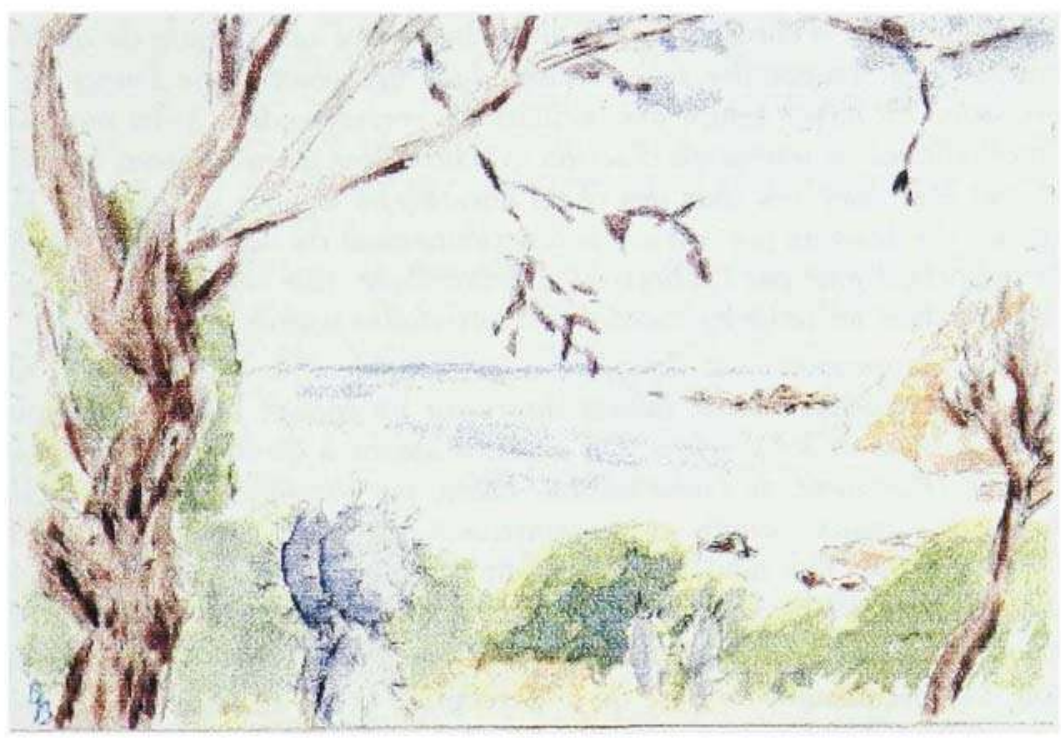

View from the Institut d'Études Scientifiques de Cargèse 


\section{AVANT-PROPOS}

Depuis une dizaine d'années, de nombreuses études ont porté sur la mécanique statistique et la dynamique d'automates neuronaux, basées le plus souvent sur une modélisation relativement ancienne du neurone. Mais les développements récents en Neurosciences ont considérablement modifié nos connaissances, à la fois sur le neurone et sur le traitement de l'information dans le cortex.

Les techniques d'enregistrement multi-électrodes ont permis de détecter des corrélations temporelles très précises dans des fenêtres de l'ordre de la milliseconde. En même temps des oscillations, correspondant à des émissions quasi-périodiques de potentiels d'action synchronisées à travers tout le cortex visuel, ont été observées chez des chats anesthésiés et chez des singes. Parallèlement, des travaux portant sur le fonctionnement du neurone ont souligné le rôle important joué par l'arborisation dendritique, rôle complètement passé sous silence dans les modèles classiques d'automates neuronaux.

Ces développements ont donné un essor considérable à des études, tant théoriques qu'expérimentales, faisant intervenir un codage par des configurations spatio-temporelles précises. On est ainsi amené à envisager de nouveaux modèles de traitement de l'information, basés, par exemple, sur la détection synchrone de signaux corrélés, et qui pourraient être particulièrement robustes vis-à-vis du bruit. Ces modèles pourraient conduire à des applications technologiques originales en matière de traitement de l'information et de contrôle.

L'approfondissement de ces thèmes peut être essentiel pour notre compréhension des mécanismes de base de la perception et de la cognition. Il peut aussi conduire à de nouveaux concepts dans les applications concernant la perception artificielle et la reconnaissance des formes. Jusqu'à présent les systèmes artificiels de reconnaissance des formes sont encore loin des possibilités de la vision humaine. On peut maintenant espérer que les systèmes basés sur le codage temporel par les spikes vont apporter une avancée significative dans cette direction.

Le but de cette école était d'apporter aux étudiants et aux chercheurs, tant fondamentaux qu'appliqués, un enseignement de pointe couvrant, dans ce domaine, l'ensemble des disciplines concernées (Neurosciences, Physique, Mathématiques, Théories de l'Information et du Contrôle), et de favoriser des coopérations interdisciplinaires et internationales. 
Chaque jour, et ceci pendant dix jours, quatre cours de quatre-vingt-dix minutes ont été dispensés, complètés par des sessions d'affiches, le tout dans le cadre d'une école d'été organisée du 30 Juin au 12 Juillet 1997 à l'Institut d'Études Scientifiques de Cargèse, en Corse. Cet institut esr une unité mixte (UMS 820) du Centre National de la Recherche Scientifique,de l'Université de Corte et de l'Université de Nice-Sophia-Antipolis. Il nous est agréable d'exprimer notre gratitude aux trois institutions pour leur hospitalité, à Elisabeth Dubois-Violette, Directeur de l'Institut, pour son aide constante et amicale, et à Annette Touchant, Secrétaire de l'Institut, ainsi qu'à tout le personnel de l'Institut, pour la qualité et la chaleur de leur accueil qui a joué un grand rôle dans le climat et le succès de l'Ecole.

L'Ecole a été en grande partie subventionnée par la Formation Permanente du Centre National de la Recherche Scientifique. Ce soutien a été décisif pour nous encourager à poursuivre notre projet. Nous espérions la participation de nombreux chercheurs CNRS, et regrettons qu'elle ait été si faible. Mais tous les jeunes étudiants francais provenaient d'unitś CNRS, nous espérons que les connaissances qu'ils ont acquises à Cargèse seront profitables pour eux et leurs collègues dans leurs travaux futurs.

Nous sommes très reconnaissants à la Direction Scientifique du Département Sciences pour l'Ingénieur du Centre National de la Recherche Scientifique qui a spontanément décidé de soutenir financièrement l'Ecole.

La contribution de la Direction des Recherches et Études Techniques Direction Générale de l'Armement du Ministère de la Défense francais a été très importante pour nous, que son Directeur trouve ici l'expression de notre gratitude.

Finalement il nous est très agréable de remercier la Collectivité Territoriale Corse, son Président et son Service de l'Enseignement et de la Recherche pour son aide et son soutien financier. Nous sommes sûrs de nous faire les interprètes de l'ensemble des participants en disant combien nous avons trouvé la Corse splendide et les Corses amicaux. L'ile magique a ainsi contribué en grande partie à l'esprit et à la réussite de l'Ecole .

Nous remercions tous nos collègues pour leur participation et la qualité de leurs cours. Nous espérions publier l'ensemble de ceux-ci et regrettons que certains manuscrits ne nous soient pas parvenus. Nous sommes très reconnaissants aux auteurs qui nous ont fait parvenir leur contribution et espérons que cette édition, même partielle, sera utile aux participants de l'Ecole et aux autres.

Soutien financier et bons cours sont essentiels mais pas suffisants pour assurer la réussite; c'est la participation de chacun aux discussions et activités qui a crée une bonne ambiance, nous remercions chaque étudiant pour sa con- 
tribution. L'exposition de leurs travaux par voie d'affiches a été un grand moment et a permis d'amorcer des collaborations fructueuses.

Notre souhait est que l'Ecole ait ouvert des perspectives et engendré de nouvelles collaborations interdisciplinaires et internationales.

Pour finir nous remercions les nombreuses personnes qui ont rendu possible l'organisation de cette manifestation et la publication des comptes-rendus, en particulier Jean-Bruno Erismann, Denis Patrat, Michèle Rossignol et Françoise Smagghe du Centre de Physique Théorique de Marseille.

Guy Burdet Phillipe Combe Olivier Parodi

Marseille, 1998. 


\section{PARTICIPANTS}

Francesca Acerra

Groupe de Développement

Neurocognitif

C.R.N.C. - C.N.R.S.

31, Chemin J. Aiguier

13402 MARSEILLE CEDEX 20

acerra@lnf.cnrs-mrs.fr

Masaharu Adachi

Laboratory for Artificial

Brain Systems, FRP

The Institute of Physical and

Chemical Research(RIKEN)

2-1 Hirosawa, Wako-shi

Saitama 351-01

Japan

adachi@zoo.riken.go.jp

Ad. Aertsen

Department of Neurobiology

and Biophysics

Institute Biology III

Schänzlestrasse 1

D 79104 Freiburg

Allemagne

aertsen@biologie.uni-freiburg.de

Rocio Aguilar-Chongtay

20/7 Dalgety Avenue

Edinburgh EH7 5UG

Scotland, UK

rocio@dai.ed.ac.uk
Joern Anemueller

Carl-von-Ossietzky-Universitaet

Oldenburg

Dept. of Physics

Medical Physics Group

D - 26111 Oldenburg

Germany

ane@medi.physik.uni-oldenburg.de

Omer Berat Artrun

Institute for Brain and Neural

Systems

Department of Physics,

Box 1843

Brown University

Providence RI 02912

USA

artun@ens.brown.edu

Alexander Asteroth

Institut fuer Informatik I

Universitaet Bonn

Roemerstr. 164

53117 Bonn

Germany

aster@informatik.uni-bonn.de

Thierry Ballain

LABORATOIRE TIMC

CNRS UMR 5525

UFR de Medecine

Universite Joseph Fourier

38706 LA TRONCHE

Thierry.Ballain@imag.fr 
Annette Bastian

CRNC - CNRS

31 , ch. Joseph Aiguier

13402 Marseille Cedex 20

bastian@lnf.cnrs-mrs.fr

Sander Bohte

The Netherlands Opthalmic

Research Institute

P.O.Box 12141

1100 AC Amsterdam

The Netherlands

smbohte@phys.uva.nl

Catherine Bourdet

Service de psychiatrie

Hopital Albert Chenevier (AP-HP)

94000 CRETEIL

bourdet@ext.jussieu.fr

CPT

Guy Burdet

CNRS-Luminy case 907

13288 Marseille CEDEX 9

burdet@cpt.univ-mrs.fr

Boris Burle

CRNC - CNRS

31, ch. Joseph Aiguier

13402 Marseille Cedex 20

time@Inf.cnrs-mrs.fr

CPT

Philippe Combe

CNRS-Luminy case 907

13288 Marseille CEDEX 9

combe@cpt.univ-mrs.fr
Jordi Cosp-Villela

Universitat Politecnica de

Catalunya

Dept. Enginyeria Electronica,

Edif. C4

c/Gran Capita s/n

08034 Barcelona

Spain

jcosp@eel.upc.es

Emmanuel Dauce

ONERA-CERT

2, avenue Edouard Belin

31055 Toulouse CEDEX

dauce@cert.fr

\section{Jacques Demongeot \\ TIMC-Imag}

Faculté de Médecine de Grenoble

$38700 \mathrm{La}$ Tronche

Jacques.Demongeot@imag.fr

Carolyn Drake

Laboratoire de Psychologie

Expérimentale

28 rue Serpente

75006 Paris

drake@idf.ext.jussieu.fr

Gérard Dreyfus

Laboratoire d'Électronique ESPCI

10 , rue Vauquelin

F 75005 Paris

Gerard.Dreyfus@espci.fr 
Denis Fize

Laboratoire Cerveau et Cognition

(Cerco)

133 route de Narbonne

31000 Toulouse

fize@cerco.ups-tlse.fr

Guillaume Fumanal

Neurosciences et Systemes

Sensoriels

(Physiologie Neurosensorielle)

Universite Claude Bernard

Lyon 1

69622 Villeurbanne CEDEX

fumanal@neurosens.univ-lyon1.fr

Sarah Gingell

Centre for Cognitive Science

University of Edinburgh

2, Buccleuch Place

Edinburgh, EH8 9LW

Scotland, U.K.

sarah@cogsci.ed.ac.uk

Olivier Gallet

ETIS-ENSEA URA 2235

6 avenue du Ponceau

95014 Cergy Cedex

gallet@ensea.fr

Jan Smit Gerard

Turfmarkt $88 \mathrm{a}$

2801 HB Gouda

Hollande

gerards@bart.nl
Franck Grammont

CRNC - CNRS

31, ch. Joseph Aiguier

13402 Marseille Cedex 20

cortex@lnf.cnrs-mrs.fr

Jeanny Hérault

Laboratoire TIRF

INPG

46 Av. Félix Viallet

38031 Grenoble

herault@tirf.inpg.fr

Alix Herrmann

Institute of Physiology

Bugnon 7

CH-1005 Lausanne

Suisse

alix@iphysiolhp1.unil.ch

John Hertz

Nordita

Blegdamsvej 17

DK-2100 Kopenhagen

Denmark

hertz@norsg2.nordita.dk

André Holley

Physiologie Neurosensorielle

Universite Claude Bernard Lyon 1

F 69622 Villeurbanne CEDEX

holley@neurosens.univ-lyon1.fr

Ramon Huerta

Institute for Nonlinear Science

University of California at San Diego

La Jolla, CA 92093

USA

huerta@routh.ucsd.edu 
Norbert Jankowski

Department of Computer Methods

Nicholas Copernicus University

ul. Grudziadzka 5

87-100 Torun

Poland

norbert@phys.uni.torun.pl

Bertrand Jouve

Laboratoire Cerveau et

Cognition

(Cerco)

133 route de Narbonne

31000 Toulouse

jouve@cerco.ups-tlse.fr

Christian Kurrer

Centre de Physique Theorique

Ecole Polytechnique

91128 Palaiseau Cedex

kurrer@orphee.polytechnique.fr

$\quad$ Abderrahim Labbi
Centre Universitaire
d'Informatique
Universite de Geneve
24, Rue du General Dufour
1211 Geneve 4
Suisse
labbi@cui.unige.ch

Michel Leseure

Manufacturing Systems

Management Unit

Department of Mechanical

Engineering

The University of Sheffield

Mappin Street

Sheffield, S1 3JD

England

m.leseure@sheffield.ac.uk
Remy Lestienne

Institut des Neurosciences

9 quai St Bernard

F-75005 Paris

Remy.Lestienne@snv.jussieu.fr

Christoph von der Malsburg Institut fuer Neuroinformatik, ND $03 / 34$

Ruhr Universitaet Bochum

D - 44780 Bochum

Germany

malsburg@neuroinformatik.

ruhr-uni-bochum.de

Morgan Mangeas

Laboratoire SAMOS

Universite PARIS I

90, rue de TOLBIAC

75634 Paris CEDEX 13

Morgan.Mangeas@der.edfgdf.fr

Ruggero Milanese

Departement d'Informatique

Universite de Geneve

24, rue General-Dufour

1211 Geneve 4

Suisse

Ruggero.Milanese@cui.unige.ch

Eric Mousset

Univ. of Bern

IAM Neural Comput.Group

Neubrckstrasse 10

3012 Bern Switzerland

mousset@iam.uribe.ch 
Jean-Pierre Nadal

ENS Département de Physique

Laboratoire de Physique Statistique

24 rue Lhompond

F75231 Paris CEDEX 05

Jean-Pierre.Nadal@lps.ens.fr

Tatjana Nazir

CRNC - CNRS

31, ch. Joseph Aiguier

13402 Marseille Cedex 20

nazir@lnf.cnrs-mrs.fr

\section{Olivier Parodi}

CPT

CNRS-Luminy case 907

13288 Marseille CEDEX 9

parodi@cpt.univ-mrs.fr

Hans Ekkehard Plesser

Institut fuer Biologie II

RWTH Aachen

D 52056 Aachen

Germany

plesser@tyto.bio2.rwth-aachen.de

Andrei Popescu-Belis

LIMSI - CNRS

Groupe Langage et Cognition

BP 133

91403 ORSAY Cedex

popescu@limsi.fr

\section{Brigitte Quenet}

Laboratoire d'Électronique

ESPCI

10 , rue Vauquelin

F 75005 Paris

Brigitte.Quenet@espci.fr
Gilles Renversez

CPT

CNRS-Luminy case 907

13288 Marseille CEDEX 9

renverse@cptsu2.univ-mrs.fr

Arnaud Rey

CRNC - CNRS

31, ch. Joseph Aiguier

13402 Marseille Cedex 20

frog@lnf.cnrs-mrs.fr

Alexa Riehle

CRNC - CNRS

31, ch. Joseph Aiguier

13402 Marseille Cedex 20

ariehle@Inf.cnrs-mrs.fr

Sergi Ripoll

Departament de Fisica

Fonamental

Facultat de Fisica,

Universitat de Barcelona

Av. Diagonal, 647

Barcelona 08028

SPAIN

sripoll@ulyses.ffn.ub.es

Pieter Roelfsema

MPI Brain Research

Deutschordenstrasse 1

D 79104 Freiburg

Allemagne

roelfsema@mpih-frankfurt.mpg.de

Michele Rossignol CPT

CNRS-Luminy case 907

13288 Marseille CEDEX 9

michele@cpt.univ-mrs.fr 
Thomas Schanze

Philipps-Universitaet

FB Physik

AG Neurophysik

Renthof 7

D-35032 Marburg

Th.Schanze@physik.

uni-marburg.de

Sara A. Solla

Depmt.of Physics

Northwestern Univ.

Sheridan Road 2145

Evanston IL 60208-3112

USA

SOLLA@NWU.EDU

Anne Spalanzani

CLIPS-IMAG

Campus Universite

Joseph Fourier

BP 53

38041 Grenoble Cedex 9

Anne.Spalanzani@imag.fr

Mona Spiridon

Centre MANTRA

Dept. d'Informatique

1015 Lausanne

Suisse

Mona.Spiridon@di.epfl.ch

David Sterratt

Centre for Cognitive Science

University of Edinburgh

2 Buccleuch Place

Edinburgh, EH8 9LW

SCOTLAND, U.K.

David.C.Sterratt@ed.ac.uk
Katrin Suder

Dept. Neurophysiology

(MA 4/149)

Ruhr Universitaet Bochum

Universitaetsstr. 15

D-44780 Bochum

Germany

suder@neurop2.

ruhr-uni-bochum.de

Enric Claverol Tinture

St/ Mare de Deu de Nuria 3 3er 2a

BARCELONA 08017

SPAIN

claverol@eel.upc.es

Jochen Triesch

Institut fuer Neuroinformatik

Ruhr-Universitaet Bochum

Universitaetsstrasse 150

D-44801 Bochum Germany

jochen@neuroinformatik

.ruhr-uni-bochum.de

Antonio Turiel

Departamento de Fisica Teorica

Facultad de Ciencias

Universidad Autonoma de Madrid

28049 MADRID

Spain

amturiel@delta.ft.uam.es

Heather Woodward

Vassar College

124 Raymond Ave.

Poughkeepsie, NY 12604

USA

hewoodward@vaxsar.vassar.edu 


\section{CONTENTS}

Temporal coding with and without clocks

Codage temporel avec et sans horloge

R. Lestienne

Modeling synfire networks

Modélisation des réseaux "Synfire"

J. A. Hertz

Neuronal decoding of temporal signal

Quelles contraintes le fonctionnement des neurones

apporte-t-il au codage et au décodage de l'information

O. Parodi

Algorithms for the detection of connectedness and their neural implementation

Algorithmes pour la détection de la connectivité et leur implementation neuronale

P. R. Roelfsema, S. Bohte, H. Spekreijse

From complex signal to adapted behavior. A theoretical approach of the honeybee olfactory brain

Du signal complexe au comportement adaptaté. Approche théorique du système olfactif de l'abeille

B. Quenet, G. Dreyfus, C. Masson

Reducing the complexity of neural nets for industrial applications and biological models

Réduction de la complexité des réseaux de neurones

pour des applications industrielles et pour des modèle biologiques

G. Dreyfus

Positive regulation circuits and memory

Circuits de régulation positifs et mémoire

J. Demongeot 
Sensory coding: information maximization and redundancy reduction

Codage sensoriel: maximisation de l'information et réduction des redondances

J.-P. Nadal, N. Parga

Learning: a geometrical approach

Une approche géométrique de l'apprentissange

G. Burdet, Ph. Combe, H. Nencka 